\title{
PBW THEOREMS AND FROBENIUS STRUCTURES FOR QUANTUM MATRICES
}

\author{
FABIO GAVARINI \\ Università di Roma "Tor Vergata" - Dipartimento di Matematica \\ Via della Ricerca Scientifica 1, I-00133 Roma-ITALY \\ e-mail:gavarini@mat.uniromal.
}

(Received 2 November, 2006; revised 31 March, 2007; accepted 24 May, 2007)

\begin{abstract}
Let $G \in\left\{\operatorname{Mat}_{n}(\mathbb{C}), G L_{n}(\mathbb{C}), S L_{n}(\mathbb{C})\right\}$, let $\mathcal{O}_{q}(G)$ be the quantum function algebra - over $\mathbb{Z}\left[q, q^{-1}\right]$ - associated to $G$, and let $\mathcal{O}_{\varepsilon}(G)$ be the specialisation of the latter at a root of unity $\varepsilon$, whose order $\ell$ is odd. There is a quantum Frobenius morphism that embeds $\mathcal{O}(G)$, the function algebra of $G$, in $\mathcal{O}_{\varepsilon}(G)$ as a central Hopf subalgebra, so that $\mathcal{O}_{\varepsilon}(G)$ is a module over $\mathcal{O}(G)$. When $G=S L_{n}(\mathbb{C})$, it is known by [3], [4] that (the complexification of) such a module is free, with $\operatorname{rank} \ell^{\operatorname{dim}(G)}$. In this note we prove a PBW-like theorem for $\mathcal{O}_{q}(G)$, and we show that - when $G$ is $M_{a t}$ or $G L_{n}$ - it yields explicit bases of $\mathcal{O}_{\varepsilon}(G)$ over $\mathcal{O}(G)$. As a direct application, we prove that $\mathcal{O}_{\varepsilon}\left(G L_{n}\right)$ and $\mathcal{O}_{\varepsilon}\left(M_{n}\right)$ are free Frobenius extensions over $\mathcal{O}\left(G L_{n}\right)$ and $\mathcal{O}\left(M_{n}\right)$, thus extending some results of [5].
\end{abstract}

2000 Mathematics Subject Classification. Primary 20G42. Secondary 81R50.

1. The general setup. Let $G$ be a complex semisimple, connected, simply connected affine algebraic group. One can introduce a quantum function algebra $\mathcal{O}_{q}(G)$, a Hopf algebra over the ground ring $\mathbb{C}\left[q, q^{-1}\right]$, where $q$ is an indeterminate, as in [7]. If $\varepsilon$ is any root of 1 , one can specialize $\mathcal{O}_{q}(G)$ at $q=\varepsilon$, which means taking the Hopf $\mathbb{C}$-algebra $\mathcal{O}_{\varepsilon}(G):=\mathcal{O}_{q}(G) /(q-\varepsilon) \mathcal{O}_{q}(G)$. In particular, for $\varepsilon=1$ one has $\mathcal{O}_{1}(G) \cong \mathcal{O}(G)$, the classical (commutative) function algebra over $G$. Moreover, if the order $\ell$ of $\varepsilon$ is odd, then there exists a Hopf algebra monomorphism $\mathfrak{F r}: \mathcal{O}(G) \cong$ $\mathcal{O}_{1}(G) \longleftrightarrow \mathcal{O}_{\varepsilon}(G)$, called quantum Frobenius morphism for $G$, which embeds $\mathcal{O}(G)$ inside $\mathcal{O}_{\varepsilon}(G)$ as a central Hopf subalgebra. Therefore, $\mathcal{O}_{\varepsilon}(G)$ is naturally a module over $\mathcal{O}(G)$. It is proved in [4] and in [3] that such a module is free, with rank $\ell^{\operatorname{dim}(G)}$. In the special case of $G=S L_{2}$, a stronger result was given in [8], where an explicit basis was found. We shall give similar results when $G$ is $G L_{n}$ or $M_{n}:=M a t_{n}$; namely we provide explicit bases of $\mathcal{O}_{\varepsilon}(G)$ as a free module over $\mathcal{O}(G)$, where in addition everything is defined replacing $\mathbb{C}$ with $\mathbb{Z}$. The proof is via some (more or less known) PBW theorems for $\mathcal{O}_{q}\left(M_{n}\right)$ and $\mathcal{O}_{q}\left(G L_{n}\right)$ - and $\mathcal{O}_{q}\left(S L_{n}\right)$ as well - as modules over $\mathbb{Z}\left[q, q^{-1}\right]$.

Let $M_{n}:=\operatorname{Mat}_{n}(\mathbb{C})$. The algebra $\mathcal{O}\left(M_{n}\right)$ of regular functions on $M_{n}$ is the unital associative commutative $\mathbb{C}$-algebra with generators $\bar{t}_{i, j}(i, j=1, \ldots, n)$. The semigroup structure on $M_{n}$ yields on $\mathcal{O}\left(M_{n}\right)$ the natural bialgebra structure given by matrix product - see [6], Ch. 7. We can also consider the semigroup-scheme $\left(M_{n}\right)_{\mathbb{Z}}$ associated to $M_{n}$, for which a like analysis applies: in particular, its function algebra $\mathcal{O}^{\mathbb{Z}}\left(M_{n}\right)$ is a $\mathbb{Z}$-bialgebra, with the same presentation as $\mathcal{O}\left(M_{n}\right)$ but over the ring $\mathbb{Z}$. 
Now we define quantum function algebras. Let $R$ be any commutative ring with unity, and let $q \in R$ be invertible. We define $\mathcal{O}_{q}^{R}\left(M_{n}\right)$ as the unital associative $R$-algebra with generators $t_{i, j}(i, j=1, \ldots, n)$ and relations

$$
\begin{aligned}
& t_{i, j} t_{i, k}=q t_{i, k} t_{i, j}, \quad t_{i, k} t_{h, k}=q t_{h, k} t_{i, k} \quad \forall j<k, i<h, \\
& t_{i, l} t_{j, k}=t_{j, k} t_{i, l}, \quad t_{i, k} t_{j, l}-t_{j, l} t_{i, k}=\left(q-q^{-1}\right) t_{i, l} t_{j, k} \quad \forall \quad i<j, k<l .
\end{aligned}
$$

It is known that $\mathcal{O}_{q}^{R}\left(M_{n}\right)$ is a bialgebra, but we do not need this extra structure in the present work (see [6] for further details - cf. also [1] and [12]).

As to specialisations, set $\mathbb{Z}_{q}:=\mathbb{Z}\left[q, q^{-1}\right]$, let $\ell \in \mathbb{N}_{+}$be odd, let $\phi_{\ell}(q)$ be the $\ell$-th cyclotomic polynomial in $q$, and let $\varepsilon:=\bar{q} \in \mathbb{Z}_{\varepsilon}:=\mathbb{Z}_{q} /\left(\phi_{\ell}(q)\right)$, so that $\varepsilon$ is a (formal) primitive $\ell$-th root of 1 in $\mathbb{Z}_{\varepsilon}$. Then

$$
\mathcal{O}_{\varepsilon}^{\mathbb{Z}_{\varepsilon}}\left(M_{n}\right)=\mathcal{O}_{q}^{\mathbb{Z}_{q}}\left(M_{n}\right) /\left(\phi_{\ell}(q)\right) \mathcal{O}_{q}^{\mathbb{Z}_{q}}\left(M_{n}\right) \cong \mathbb{Z}_{\varepsilon} \otimes_{\mathbb{Z}} \mathcal{O}_{q}^{\mathbb{Z}_{q}}\left(M_{n}\right) .
$$

It is also known that there is a bialgebra isomorphism

$$
\mathcal{O}_{1}^{\mathbb{Z}}\left(M_{n}\right) \cong \mathcal{O}_{q}^{\mathbb{Z}_{q}}\left(M_{n}\right) /(q-1) \mathcal{O}_{q}^{\mathbb{Z}_{q}}\left(M_{n}\right) \longleftrightarrow \mathcal{O}^{\mathbb{Z}}\left(M_{n}\right), \quad t_{i, j} \bmod (q-1) \mathcal{O}_{q}^{\mathbb{Z}_{q}}\left(M_{n}\right) \mapsto \bar{t}_{i, j}
$$

and a bialgebra monomorphism, called quantum Frobenius morphism ( $\varepsilon$ and $\ell$ as above),

$$
\mathfrak{F r}_{\mathbb{Z}}: \mathcal{O}^{\mathbb{Z}}\left(M_{n}\right) \cong \mathcal{O}_{1}^{\mathbb{Z}}\left(M_{n}\right) \smile \mathcal{O}_{\varepsilon}^{\mathbb{Z}}\left(M_{n}\right),\left.\quad \bar{t}_{i, j} \mapsto t_{i, j}^{\ell}\right|_{q=\varepsilon}
$$

whose image is central in $\mathcal{O}_{\varepsilon}^{\mathbb{Z}}\left(M_{n}\right)$. Thus $\mathcal{O}^{\mathbb{Z}_{\varepsilon}}\left(M_{n}\right):=\mathbb{Z}_{\varepsilon} \otimes_{\mathbb{Z}} \mathcal{O}^{\mathbb{Z}}\left(M_{n}\right)$ becomes identified - via $\mathfrak{F r}_{\mathbb{Z}}$, which clearly extends to $\mathcal{O}^{\mathbb{Z}}\left(M_{n}\right)$ by scalar extension - with a central subbialgebra of $\mathcal{O}_{\varepsilon}^{\mathbb{Z}_{\varepsilon}}\left(M_{n}\right)$, so the latter can be seen as an $\mathcal{O}^{\mathbb{Z}_{\varepsilon}}\left(M_{n}\right)$-module. By the result in [4] and [3] mentioned above, we can expect this module to be free, with $\operatorname{rank} \ell^{n^{2}}$.

All the previous framework also extends to $G L_{n}$ and to $S L_{n}$ instead of $M_{n}$. Indeed, consider the quantum determinant $D_{q}:=\sum_{\sigma \in \mathcal{S}_{n}}(-q)^{\ell(\sigma)} t_{1, \sigma(1)} t_{2, \sigma(2)} \cdots t_{n, \sigma(n)} \in$ $\mathcal{O}_{q}^{R}\left(M_{n}\right)$, where $\ell(\sigma)$ denotes the length of any permutation $\sigma$ in the symmetric group $\mathcal{S}_{n}$. Then $D_{q}$ belongs to the centre of $\mathcal{O}_{q}^{R}\left(M_{n}\right)$, hence one can extend $\mathcal{O}_{q}^{R}\left(M_{n}\right)$ by a formal inverse to $D_{q}$, i.e. defining the algebra $\mathcal{O}_{q}^{R}\left(G L_{n}\right):=\mathcal{O}_{q}^{R}\left(M_{n}\right)\left[D_{q}^{-1}\right]$. Similarly, we can define also $\mathcal{O}_{q}^{R}\left(S L_{n}\right):=\mathcal{O}_{q}^{R}\left(M_{n}\right) /\left(D_{q}-1\right)$. Now $\mathcal{O}_{q}^{R}\left(G L_{n}\right)$ and $\mathcal{O}_{q}^{R}\left(S L_{n}\right)$ are Hopf $R$-algebras, and the maps $\mathcal{O}_{q}^{R}\left(M_{n}\right) \longleftrightarrow \mathcal{O}_{q}^{R}\left(G L_{n}\right), \mathcal{O}_{q}^{R}\left(G L_{n}\right) \longrightarrow \mathcal{O}_{q}^{R}\left(S L_{n}\right)$, $\mathcal{O}_{q}^{R}\left(M_{n}\right) \longrightarrow \mathcal{O}_{q}^{R}\left(S L_{n}\right)$ (the third one being the composition of the first two) given by $t_{i, j} \mapsto t_{i, j}$ are epimorphisms of $R$-bialgebras, and even of Hopf $R$-algebras in the second case. The specialisations

$$
\begin{aligned}
& \mathcal{O}_{\varepsilon}^{\mathbb{Z}_{\varepsilon}}\left(G L_{n}\right)=\mathcal{O}_{q}^{\mathbb{Z}_{q}}\left(G L_{n}\right) /\left(\phi_{\ell}(q)\right) \mathcal{O}_{q}^{\mathbb{Z}_{q}}\left(G L_{n}\right) \cong \mathbb{Z}_{\varepsilon} \otimes_{\mathbb{Z}} \mathcal{O}_{q}^{\mathbb{Z}_{q}}\left(G L_{n}\right) \\
& \mathcal{O}_{\varepsilon}^{\mathbb{Z}_{\varepsilon}}\left(S L_{n}\right)=\mathcal{O}_{q}^{\mathbb{Z}_{q}}\left(S L_{n}\right) /\left(\phi_{\ell}(q)\right) \mathcal{O}_{q}^{\mathbb{Z}_{q}}\left(S L_{n}\right) \cong \mathbb{Z}_{\varepsilon} \otimes_{\mathbb{Z}} \mathcal{O}_{q}^{\mathbb{Z}_{q}}\left(S L_{n}\right)
\end{aligned}
$$

enjoy the same properties as above, namely there exist isomorphisms $\mathcal{O}_{1}^{\mathbb{Z}}\left(G L_{n}\right) \cong$ $\mathcal{O}^{\mathbb{Z}}\left(G L_{n}\right)$ and $\mathcal{O}_{1}^{\mathbb{Z}}\left(S L_{n}\right) \cong \mathcal{O}^{\mathbb{Z}}\left(S L_{n}\right)$ and there are quantum Frobenius morphisms

$$
\begin{aligned}
& \mathfrak{F r}_{\mathbb{Z}}: \mathcal{O}^{\mathbb{Z}}\left(G L_{n}\right) \cong \mathcal{O}_{1}^{\mathbb{Z}}\left(G L_{n}\right) \longleftrightarrow \mathcal{O}_{\varepsilon}^{\mathbb{Z}}\left(G L_{n}\right), \\
& \mathfrak{F r}_{\mathbb{Z}}: \mathcal{O}^{\mathbb{Z}}\left(S L_{n}\right) \cong \mathcal{O}_{1}^{\mathbb{Z}}\left(S L_{n}\right) \longleftrightarrow \mathcal{O}_{\varepsilon}^{\mathbb{Z}_{\varepsilon}}\left(S L_{n}\right)
\end{aligned}
$$


described by the same formulæ as for $M_{n}$. Moreover, $D_{q}^{ \pm 1} \bmod (q-1) \mapsto D^{ \pm 1}$ in the isomorphisms and $D^{ \pm 1} \cong D_{q}^{ \pm 1} \bmod (q-1) \mapsto D_{q}^{ \pm \ell} \bmod (q-\varepsilon)$ in the quantum Frobenius morphisms for $G L_{n}$ (which extend those of $M_{n}$ ). In addition, all these isomorphisms and quantum Frobenius morphisms are compatible (in the obvious sense) with the natural maps which link $\mathcal{O}_{q}^{\mathbb{Z}_{q}}\left(M_{n}\right), \mathcal{O}_{q}^{\mathbb{Z}_{q}}\left(G L_{n}\right)$ and $\mathcal{O}_{q}^{\mathbb{Z}_{q}}\left(S L_{n}\right)$, and their specialisations, to each other.

Like for $M_{n}$, the image of the quantum Frobenius morphisms are central in $\mathcal{O}_{\varepsilon}^{\mathbb{Z}_{\varepsilon}}\left(G L_{n}\right)$ and in $\mathcal{O}_{\varepsilon}^{\mathbb{Z}_{\varepsilon}}\left(S L_{n}\right)$. Thus $\mathcal{O}^{\mathbb{Z}_{\varepsilon}}\left(G L_{n}\right):=\mathbb{Z}_{\varepsilon} \otimes_{\mathbb{Z}} \mathcal{O}^{\mathbb{Z}}\left(G L_{n}\right)$ identifies to a central Hopf subalgebra of $\mathcal{O}_{\varepsilon}^{\mathbb{Z}_{\varepsilon}}\left(G L_{n}\right)$, and $\mathcal{O}^{\mathbb{Z}_{\varepsilon}}\left(S L_{n}\right):=\mathbb{Z}_{\varepsilon} \otimes_{\mathbb{Z}} \mathcal{O}^{\mathbb{Z}}\left(S L_{n}\right)$ identifies to a central Hopf subalgebra of $\mathcal{O}_{\varepsilon}^{\mathbb{Z}}\left(S L_{n}\right)$; so $\mathcal{O}_{\varepsilon}^{\mathbb{Z}}\left(G L_{n}\right)$ is an $\mathcal{O}^{\mathbb{Z}}\left(G L_{n}\right)$-module and $\mathcal{O}_{\varepsilon}^{\mathbb{Z}}\left(S L_{n}\right)$ is an $\mathcal{O}^{\mathbb{Z}}\left(S L_{n}\right)$-module.

In $\S 2$, we shall prove (Theorem 2.1) a PBW-like theorem providing several different bases for $\mathcal{O}_{q}^{R}\left(M_{n}\right), \mathcal{O}_{q}^{R}\left(G L_{n}\right)$ and $\mathcal{O}_{q}^{R}\left(S L_{n}\right)$ as $R$-modules. As an application, we find (Theorem 2.2) explicit bases of $\mathcal{O}_{\varepsilon}^{\mathbb{Z}_{\varepsilon}}\left(M_{n}\right)$ as an $\mathcal{O}^{\mathbb{Z}_{\varepsilon}}\left(M_{n}\right)$-module, which then in particular is free of rank $\ell^{\operatorname{dim}\left(M_{n}\right)}$. The same bases are also $\mathcal{O}^{\mathbb{Z}_{\varepsilon}}\left(G L_{n}\right)$-bases for $\mathcal{O}_{\varepsilon}^{\mathbb{Z}_{\varepsilon}}\left(G L_{n}\right)$, which then is free of rank $\ell^{\operatorname{dim}\left(G L_{n}\right)}$. Both results can be seen as extensions of some results in [4].

Finally, in $\S 3$ we use the above mentioned bases to prove that $\mathcal{O}^{\mathbb{Z}_{\varepsilon}}\left(M_{n}\right)$ is a free Frobenius extension of its central subalgebra $\mathcal{O}^{\mathbb{Z}_{\varepsilon}}\left(M_{n}\right)$, and to explicitly compute the associated Nakayama automorphism. The same we do for $\mathcal{O}_{\varepsilon}^{\mathbb{Z}_{\varepsilon}}\left(G L_{n}\right)$ as well. Everything follows from the ideas and methods in [5], now applied to the explicit bases given by Theorem 2.2 .

\section{PBW-like theorems.}

THEOREM 2.1. ( $P B W$ theorem for $\mathcal{O}_{q}^{R}\left(M_{n}\right), \mathcal{O}_{q}^{R}\left(G L_{n}\right)$ and $\mathcal{O}_{q}^{R}\left(S L_{n}\right)$ as $R$-modules) Assume $(q-1)$ is not invertible in $R_{q}:=\left\langle q, q^{-1}\right\rangle$, the subring of $R$ generated by $q$ and $q^{-1}$.

(a) Let any total order be fixed in $\{1, \ldots, n\}^{\times 2}$. Then the following sets of ordered monomials are $R$-bases of $\mathcal{O}_{q}^{R}\left(M_{n}\right)$, resp. $\mathcal{O}_{q}^{R}\left(G L_{n}\right)$, resp. $\mathcal{O}_{q}^{R}\left(S L_{n}\right)$, as modules over $R$ :

$$
\begin{aligned}
B_{M} & :=\left\{\prod_{i, j=1}^{n} t_{i, j}^{N_{i, j}} \mid N_{i, j} \in \mathbb{N} \forall i, j\right\} \\
B_{G L}^{\wedge} & :=\left\{\prod_{i, j=1}^{n} t_{i, j}^{N_{i, j}} D_{q}^{-N} \mid N, N_{i, j} \in \mathbb{N} \forall i, j ; \min \left(\left\{N_{i, i}\right\}_{1 \leq i \leq n} \cup\{N\}\right)=0\right\} \\
B_{G L}^{\vee} & :=\left\{\prod_{i, j=1}^{n} t_{i, j}^{N_{i, j}} D_{q}^{Z} \mid Z \in \mathbb{Z}, N_{i, j} \in \mathbb{N} \forall i, j ; \min \left\{N_{i, i}\right\}_{1 \leq i \leq n}=0\right\} \\
B_{S L} & :=\left\{\prod_{i, j=1}^{n} t_{i, j}^{N_{i, j}} \mid N_{i, j} \in \mathbb{N} \forall i, j ; \min \left\{N_{i, i}\right\}_{1 \leq i \leq n}=0\right\} .
\end{aligned}
$$

(b) Let $\preceq$ be any total order fixed in $\{1, \ldots, n\}^{\times 2}$ such that $(i, j) \preceq(h, k) \preceq(l, m)$ whenever $j>n+1-i, k=n+1-h, m<n+1-l$. Then the following sets of ordered 
monomials are $R$-bases of $\mathcal{O}_{q}^{R}\left(G L_{n}\right)$, resp. $\mathcal{O}_{q}^{R}\left(S L_{n}\right)$, as modules over $R$ :

$$
\begin{aligned}
B_{G L}^{\wedge,-} & :=\left\{\prod_{i, j=1}^{n} t_{i, j}^{N_{i, j}} D_{q}^{-N} \mid N, N_{i, j} \in \mathbb{N} \forall i, j ; \min \left(\left\{N_{i, n+1-i}\right\}_{1 \leq i \leq n} \cup\{N\}\right)=0\right\} \\
B_{G L}^{\vee,-} & :=\left\{\prod_{i, j=1}^{n} t_{i, j}^{N_{i, j}} D_{q}^{Z} \mid Z \in \mathbb{Z}, N_{i, j} \in \mathbb{N} \forall i, j ; \min \left\{N_{i, n+1-i}\right\}_{1 \leq i \leq n}=0\right\} \\
B_{S L}^{-} & :=\left\{\prod_{i, j=1}^{n} t_{i, j}^{N_{i, j}} \mid N_{i, j} \in \mathbb{N} \forall i, j ; \min \left\{N_{i, n+1-i}\right\}_{1 \leq i \leq n}=0\right\} .
\end{aligned}
$$

Proof. Roughly speaking, our method is a (partial) application of the diamond lemma (see [2]): however, we do not follow it in all details, as we use a specialisation trick as a shortcut.

If we prove our results for the algebras defined over $R_{q}$ instead of $R$, then the same results will hold as well by scalar extension. Thus we can assume $R=R_{q}$, and then we note that, by our assumption, the specialised $\operatorname{ring} \bar{R}:=R /(q-1) R \neq\{0\}$ is non-trivial.

Proof of $(a)$ : (see also [10], Theorem 3.1, and [12], Theorem 3.5.1)

We begin with $\mathcal{O}_{q}^{R}\left(M_{n}\right)$. It is clearly spanned over $R$ by the set of all (possibly unordered) monomials in the $t_{i j}$ 's: so we must only prove that any such monomial belongs to the $R$-span of the ordered monomials. In fact, the latter are linearly independent, since such are their images via specialisation $\mathcal{O}_{q}^{R}\left(M_{n}\right) \longrightarrow \mathcal{O}_{q}^{R}\left(M_{n}\right) /(q-$ $1) \mathcal{O}_{q}^{R}\left(M_{n}\right) \cong \mathcal{O}_{1}^{\bar{R}}\left(M_{n}\right)$.

Thus, take any (possibly unordered) monomial in the $t_{i j}$ 's, say $\underline{t}:=t_{i_{1}, j_{i}} t_{i_{2}, j_{2}} \cdots t_{i_{k}, j_{k}}$, where $k$ is the degree of $t$ : we associate to it its weight, defined as

$$
w(\underline{t}):=\left(k, d_{1,1}, d_{1,2}, \ldots, d_{1, n}, d_{2,1}, d_{2,2}, \ldots, d_{2, n}, d_{3,1}, \ldots, d_{n-1, n}, d_{n, 1}, d_{n, 2}, \ldots, d_{n, n}\right)
$$

where $d_{i, j}:=\left|\left\{s \in\{1, \ldots, k\} \mid\left(i_{s}, j_{s}\right)=(i, j)\right\}\right|=$ number of occurrences of $t_{i, j}$ in $\underline{t}$. Then $w(t) \in \mathbb{N}^{n^{2}+1}$, and we consider $\mathbb{N}^{n^{2}+1}$ as a totally ordered set with respect to the (total) lexicographic order $\leq_{l e x}$. By a quick look at the defining relations of $\mathcal{O}_{q}^{R}\left(M_{n}\right)$, namely

$$
\begin{array}{rlrl}
t_{i, j} t_{i, k}=q t_{i, k} t_{i, j}, \quad t_{i, k} t_{h, k} & =q t_{h, k} t_{i, k} & & j<k, i<h, \\
t_{i, l} t_{j, k}=t_{j, k} t_{i, l}, \quad t_{i, k} t_{j, l}-t_{j, l} t_{i, k} & =\left(q-q^{-1}\right) t_{i, l} t_{j, k} & \forall \quad i<j, k<l .
\end{array}
$$

one easily sees that the weight defines an algebra filtration on $\mathcal{O}_{q}^{R}\left(M_{n}\right)$.

Now, using these same relations, one can re-order the $t_{i j}$ 's in any monomial according to the fixed total order. During this process, only two non-trivial things may occur, namely:

-1) some powers of $q$ show up as coefficients (when a relation in the first line is employed);

-2) a new summand is added (when the bottom-right relation is used);

If only steps of type 1) occur, then the process eventually stops with an ordered monomial in the $t_{i j}$ 's multiplied by a power of $q$. Whenever instead a step of type 2) occurs, the newly added term is just a coefficient $\left(q-q^{-1}\right)$ times a (possibly unordered) monomial in the $t_{i j}$ 's, call it $\underline{t}^{\prime}$ : however, by construction $w\left(\underline{t}^{\prime}\right) \supsetneqq l e x w(\underline{t})$. Then, by induction on the weight, we can assume that $\underline{t}^{\prime}$ lies in the $R$-span of the ordered 
monomials, so we can ignore the new summand. The process stops in finitely many steps, and we are done with $\mathcal{O}_{q}^{R}\left(M_{n}\right)$.

Second, we look at $\mathcal{O}_{q}^{R}\left(G L_{n}\right)$. Let us consider $f \in \mathcal{O}_{q}^{R}\left(G L_{n}\right)$. By definition, there exists $N \in \mathbb{N}$ such that $f D_{q}^{N} \in \mathcal{O}_{q}^{R}\left(M_{n}\right)$; therefore, by the result for $\mathcal{O}_{q}^{R}\left(M_{n}\right)$ just proved, we can expand $f D_{q}^{N}$ as an $R$-linear combination of ordered monomials, call them $\underline{t}=\prod_{i, j=1}^{n} t_{i, j}^{N_{i, j}}$. Thus, $f$ itself is an $R$-linear combination of monomials $\underline{t} D_{q}^{-N}$, so the latter span $\mathcal{O}_{q}^{R}\left(G L_{n}\right)$.

Now consider an ordered monomial $\underline{t}=\prod_{i, j=1}^{n} t_{i, j}^{N_{i, j}}$ in which $N_{i, i}>0$ for all $i$. Then we can re-arrange the $t_{i, i}$ 's in $\underline{t}$ so to single out a factor $t_{1,1} t_{2,2} \cdots t_{n-1, n-1} t_{n, n}$, up to "paying the cost" (perhaps) of producing some new summands of lower weight: the outcome reads

$$
\underline{t}=q^{s} \underline{t}_{0} t_{1,1} t_{2,2} \cdots t_{n-1, n-1} t_{n, n}+\text { l.t.'s }
$$

for some $s \in \mathbb{Z}$, with $\underline{t}_{0}:=\prod_{i, j=1}^{n} t_{i, j}^{N_{i, j}-\delta_{i, j}}$ having lower weight than $\underline{t}$, and the expression l.t.'s standing for an $R$-linear combination of some monomials $\underline{\underline{t}}$ such that $w(\underline{t}) \supsetneqq$ lex $w(t)$. Then we re-write the monomial $t_{1,1} t_{2,2} \cdots t_{n-1, n-1} t_{n, n}$ using the identity

$$
t_{1,1} t_{2,2} \cdots t_{n-1, n-1} t_{n, n}=D_{q}-\sum_{\substack{\sigma \in \mathcal{S}_{n} \\ \sigma \neq i d}}(-q)^{\ell(\sigma)} t_{1, \sigma(1)} t_{2, \sigma(2)} \cdots t_{n, \sigma(n)}=D_{q}+\text { l.t.'s }
$$

and we replace the right-hand side of (2.2) inside (2.1). We get $\underline{t}=q^{s} \underline{t}_{0} D_{q}+$ l.t.'s (for $D_{q}$ is central!), where now $\underline{t}_{0}$ and all monomials within l.t.'s have strictly lower weight than $\underline{t}$.

If we look now at $\underline{t} D_{q}^{z}$ (for some $z \in \mathbb{Z}$ ), we can re-write $\underline{t}$ as above, thus getting

$$
\underline{t} D_{q}^{z}=q^{s} \underline{t}_{0} D_{q} D_{q}^{z}+\text { l.t.'s }=q^{s} \underline{t}_{0} D_{q}^{z+1}+\text { l.t.'s }
$$

where l.t.'s is an $R$-linear combination of monomials $\underline{\tilde{t}} D_{q}^{z+1}$ such that $w(\underline{t}) \supsetneqq l e x w(\underline{t})$.

By repeated use of (2.3) as a reduction argument, we can easily show - by induction on the weight - that any monomial of type $\underline{t} D_{q}^{-N}(N \in \mathbb{N})$ can be expanded as an $R$ linear combination of elements of $B_{G L}^{\wedge}$ or elements of $B_{G L}^{\vee}$. Thus, both these sets do $\operatorname{span} \mathcal{O}_{q}^{R}\left(G L_{n}\right)$.

To finish with, both $B_{G L}^{\wedge}$ and $B_{G L}^{\vee}$ are $R$-linearly independent, as their image through the specialisation epimorphism $\mathcal{O}_{q}^{R}\left(G L_{n}\right) \longrightarrow \mathcal{O}_{1}^{\bar{R}}\left(G L_{n}\right) \cong \mathcal{O}^{\bar{R}}\left(G L_{n}\right)$ are $\bar{R}$ bases of $\mathcal{O}^{\bar{R}}\left(G L_{n}\right)$.

As to $\mathcal{O}_{q}^{R}\left(S L_{n}\right)$, we can repeat the argument for $\mathcal{O}_{q}^{R}\left(G L_{n}\right)$. First, $B_{S L}$ is linearly independent, for its image through specialisation $\mathcal{O}_{q}^{R}\left(S L_{n}\right) \longrightarrow \mathcal{O}_{1}^{\bar{R}}\left(S L_{n}\right) \cong \mathcal{O}^{\bar{R}}\left(S L_{n}\right)$ is an $\bar{R}$-basis of $\mathcal{O}^{\bar{R}}\left(S L_{n}\right)$. Second, the epimorphism $\mathcal{O}_{q}^{R}\left(M_{n}\right) \longrightarrow \mathcal{O}_{q}^{R}\left(S L_{n}\right)\left(t_{i, j} \mapsto t_{i, j}\right)$, and the result for $\mathcal{O}_{q}^{R}\left(M_{n}\right)$, imply that the $R$-span of $S_{S L}:=\left\{\prod_{i, j=1}^{n} t_{i, j}^{N_{i, j}} \mid N_{i, j} \in \mathbb{N} \forall i, j\right\}$ is $\mathcal{O}_{q}^{R}\left(S L_{n}\right)$. Thus one is only left to prove that each monomial $\underline{t}=\prod_{i, j=1}^{n} t_{i, j}^{N_{i, j}} \in S_{S L}$ belongs to the $R$-span of $B_{S L}$ : as before, this can be done by induction on the weight, using the reduction formula $\underline{t}=q^{s} \underline{t}_{0} D_{q}+$ l.t.'s (see above), and plugging into the relation $D_{q}=1$. 
Alternatively, we recall there is an isomorphism $\mathcal{O}_{q}^{R}\left(S L_{n}\right) \otimes_{R} R\left[x, x^{-1}\right] \cong$ $\mathcal{O}_{q}^{R}\left(G L_{n}\right)$ (of $R$-algebras) given by $t_{i, j} \otimes x^{z} \mapsto D_{q}^{-\delta_{i, 1}} t_{i, j} \cdot D_{q}^{z}$ (cf. [11]). This along with the result about $B_{G L}^{\vee}$ clearly implies that also $B_{S L}$ is an $R$-basis for $\mathcal{O}_{q}^{R}\left(S L_{n}\right)$, as claimed.

Proof of $(b)$ : First look at $\mathcal{O}_{q}^{R}\left(G L_{n}\right)$. If $f \in \mathcal{O}_{q}^{R}\left(G L_{n}\right)$, as in the proof of $(a)$ we expand $f D_{q}^{N}$ as an $R$-linear combination of ordered (according to $\preceq$ ) monomials of type $\underline{t}=\underline{t}^{-} \underline{t}^{=} \underline{t}^{+}$, with $\underline{t}^{-}:=\prod_{j>n+1-i} t_{i, j}^{N_{i, j}}, \underline{t}=\prod_{j=n+1-i} t_{i, j}^{N_{i, j}}$ and $\underline{t}^{+}:=\prod_{j<n+1-i} t_{i, j}^{N_{i, j}}$. So $f$ is an $R$-linear combination of monomials $\underline{t}^{-} \underline{t}^{=} \underline{t}^{+} D_{q}^{-N}$, hence the latter $\operatorname{span} \mathcal{O}_{q}^{R}\left(G L_{n}\right)$.

We show that each (ordered) monomial $\underline{t}^{-} \underline{t}^{=} \underline{t}^{+} D_{q}^{-N}$ belongs both to the $R$-span of $B_{G L}^{\wedge,-}$ and of $B_{G L}^{\vee,-}$, by induction on the (total) degree of the monomial $\underline{t}^{=}$. The basis of induction is $\operatorname{deg}\left(\underline{t}^{=}\right)=0$, so that $\underline{t}^{=}=1$ and $\underline{t}^{-} \underline{t}^{=} \underline{t}^{+} D_{q}^{-N}=\underline{t}^{-} \underline{t}^{+} D_{q}^{-N} \in B_{G L}^{\wedge,-} \cap B_{G L}^{\vee,-}$.

As a matter of notation, let $\mathcal{N}^{-}$, resp. $\mathcal{H}$, resp. $\mathcal{N}^{+}$, be the $R$-subalgebra of $\mathcal{O}_{q}^{R}\left(M_{n}\right)$ generated by the $t_{i, j}$ 's with $j>n+1-i$, resp. $j=n+1-i$, resp. $j<n+1-i$. Note that $\mathcal{H}$ is Abelian, and $\underline{t}^{-} \in \mathcal{N}^{-}, \underline{t}^{=} \in \mathcal{H}, \underline{t}^{+} \in \mathcal{N}^{+}$.

Now assume that all the exponents $N_{i, n+1-i}$ 's in the factor $\underline{t}^{=}$are strictly positive. As $\mathcal{H}$ is Abelian, we can draw out of $\underline{t}^{=}$(even out of $\underline{t}=\underline{t}^{-} \underline{t}^{=} \underline{t}^{+}$) a factor $t_{n, 1} t_{n-1,2} \cdots t_{2, n-1} t_{1, n}$. Now recall that $D_{q}$ can be expanded as $D_{q}=$ $\sum_{\sigma \in \mathcal{S}_{n}}(-q)^{\ell(\sigma)} t_{n, \sigma(n)} t_{n-1, \sigma(n-1)} \cdots t_{2, \sigma(2)} t_{1, \sigma(1)}$ (see, e.g., [12] or [10]). Then we can re-write the monomial $t_{n, 1} t_{n-1,2} \cdots t_{2, n-1} t_{1, n}$ as

$$
t_{n, 1} t_{n-1,2} \cdots t_{1, n}=(-q)^{-\ell\left(\sigma_{0}\right)} D_{q}-\sum_{\substack{\sigma \in \mathcal{S}_{n} \\ \sigma \neq \sigma_{0}}}(-q)^{\ell(\sigma)-\ell\left(\sigma_{0}\right)} t_{n, \sigma(n)} t_{n-1, \sigma(n-1)} \cdots t_{1, \sigma(1)}
$$

where $\sigma_{0} \in \mathcal{S}_{n}$ is the permutation $i \mapsto(n+1-i)$. Note also that we can reorder the factors in the summands of (2.4) so that all factors $t_{i, j}$ from $\mathcal{N}^{-}$are on the left of those from $\mathcal{N}^{+}$.

Now we replace the right-hand side of (2.4) in the factor $\underline{t}^{=}$within $\underline{t}=\underline{t}^{-} \underline{t}^{=} \underline{t}^{+}$, thus

$$
\underline{t}^{-} \underline{t}^{=} \underline{t}^{+}=(-q)^{-\ell\left(\sigma_{0}\right)} \underline{t}^{-} \underline{t}_{0}^{=} D_{q} \underline{t}^{+}+\text {l.t.'s }=(-q)^{-\ell\left(\sigma_{0}\right)} \underline{t}^{-} \underline{t}_{0}^{=} \underline{t}^{+} D_{q}+\text { l.t.'s. }
$$

Here $\underline{t}_{0}^{=}:=\underline{t}^{=}\left(t_{n, 1} t_{n-1,2} \cdots t_{2, n-1} t_{1, n}\right)^{-1}$ has lower (total) degree than $\underline{t}^{=}$, and the expression l.t.'s stands for an $R$-linear combination of some other monomials $\underline{t}^{-} \underline{t}^{=} \underline{t}^{+}$ (like $\underline{t}^{-} \underline{t}^{=} \underline{t}^{+}$above) in which again the degree of $\underline{t}^{=}$is lower than the degree of $\underline{t}^{=}$. In fact, this holds because when any factor $t_{i, \sigma(i)} \in \overline{\mathcal{N}}^{-}$is pulled from the right to the left of any monomial in $\underline{t}^{=} \in \mathcal{H}$ the degree of $\underline{\underline{t}}=$ is not increased. By induction on this degree, we can easily conclude that every ordered monomial $\underline{t}^{-} \underline{t}^{=} \underline{t}^{+} D_{q}^{z}$ (with $z \in \mathbb{Z}$ ) belongs to both the $R$-span of $B_{G L}^{\wedge,-}$ and the $R$-span of $B_{G L}^{\vee,-}$. That is, both sets span $\mathcal{O}_{q}^{R}\left(G L_{n}\right)$.

Eventually, both $B_{G L}^{\wedge,-}$ and $B_{G L}^{\vee,-}$ are linearly independent, as their image through the specialisation epimorphism $\mathcal{O}_{q}^{R}\left(G L_{n}\right) \longrightarrow \mathcal{O}_{1}^{\bar{R}}\left(G L_{n}\right) \cong \mathcal{O}^{\bar{R}}\left(G L_{n}\right)$ are $\bar{R}$-bases of $\mathcal{O}^{\bar{R}}\left(G L_{n}\right)$.

Second, we look at $\mathcal{O}_{q}^{R}\left(S L_{n}\right)$. As for claim $(a)$, we can repeat again - mutatis mutandis - the argument for $\mathcal{O}_{q}^{R}\left(G L_{n}\right)$, which does work again - one only has to plug in the additional relation $D_{q}=1$ too. Otherwise, as an alternative proof, we can note that the isomorphism $\mathcal{O}_{q}^{R}\left(S L_{n}\right) \otimes_{R} R\left[x, x^{-1}\right] \cong \mathcal{O}_{q}^{R}\left(G L_{n}\right)$ together with the result about $B_{G L}^{\vee,-}$ easily implies that $B_{S L}^{-}$too is an $R$-basis for $\mathcal{O}_{q}^{R}\left(S L_{n}\right)$, q.e.d. 
REMARK 2.2. (1) Claim (a) of Theorem 2.1 for $M_{n}$ only was independently proved in [12] and in [10], but taking a field as ground ring. In [10], claim (b) for $G L_{n}$ only was proved as well. Similarly, the analogue of claim $(b)$ for $S L_{n}$ only was proved in [9], $\S 7$, but taking as ground ring the field $k(q)$ - for any field $k$ of zero characteristic. Our proof then provides an alternative, unifying approach, which yields stronger results over $R$.

(2) We would better point out a special aspect of the basic assumption of Theorem 2.1 about $q$ and $R$. Namely, if the subring $\langle 1\rangle$ of $R$ generated by 1 has prime characteristic (hence it is a finite field) then the condition on $(q-1)$ is equivalent to $q$ being trascendental over $R_{q}$ or $q=1$. But if instead the characteristic of $\langle 1\rangle$ is zero or positive non-prime, then $(q-1)$ might be non-invertible in $R_{q}$ even though $q$ is algebraic (or even integral) over $\langle 1\rangle$.

The end of the story is that Theorem 2.1 holds true in the "standard" case of trascendental values of $q$, but also in more general situations.

(3) The argument used in the proof of Theorem 2.1 to get the result for $\mathcal{O}_{q}^{R}\left(S L_{n}\right)$ from those for $\mathcal{O}_{q}^{R}\left(G L_{n}\right)$, via the isomorphism $\mathcal{O}_{q}^{R}\left(S L_{n}\right) \otimes_{R} R\left[x, x^{-1}\right] \cong \mathcal{O}_{q}^{R}\left(G L_{n}\right)$, actually works both ways. Therefore, one can also prove the results $\operatorname{directly}$ for $\mathcal{O}_{q}^{R}\left(S L_{n}\right)$ - as we have sketched above - and from them deduce those for $\mathcal{O}_{q}^{R}\left(G L_{n}\right)$. Even more, as we have proved independently the results for $\mathcal{O}_{q}^{R}\left(G L_{n}\right)$ - i.e., $B_{G L}^{\vee}$ and $B_{G L}^{\vee,-}$ are $R$-bases - and for $\mathcal{O}_{q}^{R}\left(S L_{n}\right)$ - i.e., $B_{S L}$ and $B_{S L}^{-}$are $R$-bases - we can use them to prove that the algebra morphism $\mathcal{O}_{q}^{R}\left(S L_{n}\right) \otimes_{R} R\left[x, x^{-1}\right] \longrightarrow \mathcal{O}_{q}^{R}\left(G L_{n}\right)$ is in fact bijective.

(4) The orders considered in claim (b) of Theorem 2.1 refer to a triangular decomposition of $\mathcal{O}_{q}^{R}\left(G L_{n}\right)$ and $\mathcal{O}_{q}^{R}\left(S L_{n}\right)$ which is opposite to the standard one. This opposite decomposition was introduced - and its importance was especially pointed out - in [10].

We are now ready to state and prove the main result of this paper:

THEOREM 2.3. (PBW theorem for $\mathcal{O}_{\varepsilon}^{\mathbb{Z}_{\varepsilon}}(G)$ as an $\mathcal{O}^{\mathbb{Z}_{\varepsilon}}(G)$-module, for $G \in\left\{M_{n}, G L_{n}\right\}$ ) Let any total order be fixed in $\{1, \ldots, n\}^{\times 2}$. Then the set of ordered monomials

$$
\mathrm{B}_{G L}^{M}:=\left\{\prod_{i, j=1}^{n} t_{i, j}^{N_{i, j}} \mid 0 \leq N_{i, j} \leq \ell-1, \forall i, j\right\}
$$

thought of as a subset of $\mathcal{O}_{\varepsilon}^{\mathbb{Z}_{\varepsilon}}\left(M_{n}\right) \subset \mathcal{O}_{\varepsilon}^{\mathbb{Z}_{\varepsilon}}\left(G L_{n}\right)$, is a basis of $\mathcal{O}_{\varepsilon}^{\mathbb{Z}_{\varepsilon}}\left(M_{n}\right)$ as a module over $\mathcal{O}^{\mathbb{Z}_{\varepsilon}}\left(M_{n}\right)$, and a basis of $\mathcal{O}_{\varepsilon}^{\mathbb{Z}_{\varepsilon}}\left(G L_{n}\right)$ as a module over $\mathcal{O}^{\mathbb{Z}_{\varepsilon}}\left(G L_{n}\right)$.

In particular, both modules are free of rank $\ell^{\operatorname{dim}(G)}$, with $G \in\left\{M_{n}, G L_{n}\right\}$.

Proof. When specialising, Theorem 2.1(a) implies that $\mathcal{O}_{\varepsilon}^{\mathbb{Z}_{\varepsilon}}\left(M_{n}\right)$ is a free $\mathbb{Z}_{\varepsilon}$-module with $\left.B_{M}\right|_{q=\varepsilon}=\left\{\prod_{i, j=1}^{n} t_{i j}^{N_{i j}} \mid N_{i j} \in \mathbb{N} \forall i, j\right\}$ as basis - where, by abuse of notation, we write again $t_{i j}$ for $\left.t_{i j}\right|_{q=\varepsilon}$. Now, whenever the exponent $N_{i j}$ is a multiple of $\ell$, the power $t_{i j}^{N_{i j}}$ belongs to the isomorphic image $\mathfrak{F r}_{\mathbb{Z}}\left(\mathcal{O}^{\mathbb{Z}_{\varepsilon}}\left(M_{n}\right)\right)$ of $\mathcal{O}^{\mathbb{Z}_{\varepsilon}}\left(M_{n}\right)$ inside $\mathcal{O}_{\varepsilon}^{\mathbb{Z}_{\varepsilon}}\left(M_{n}\right)$, hence it is a scalar for the $\mathcal{O}^{\mathbb{Z}_{\varepsilon}}\left(M_{n}\right)$-module structure of $\mathcal{O}_{\varepsilon}^{\mathbb{Z}_{\varepsilon}}\left(M_{n}\right)$. Therefore, reducing all exponents modulo $\ell$ we find that $\mathrm{B}_{G L}^{M}$ is a spanning set for the $\mathcal{O}^{\mathbb{Z}_{\varepsilon}}\left(M_{n}\right)$-module $\mathcal{O}_{\varepsilon}^{\mathbb{Z}_{\varepsilon}}\left(M_{n}\right)$. In addition, $\mathcal{O}^{\mathbb{Z}}\left(M_{n}\right)$ clearly admits as $\mathbb{Z}$-basis the set $\bar{B}_{M}=\left\{\prod_{i, j=1}^{n} \bar{t}_{i j}^{N_{i j}} \mid N_{i j} \in \mathbb{N} \forall i, j\right\}$. It follows that $\bar{B}_{M}$ is also a $\mathbb{Z}_{\varepsilon}$-basis of $\mathcal{O}^{\mathbb{Z}_{\varepsilon}}\left(M_{n}\right)$, so $\mathfrak{F r}_{\mathbb{Z}}\left(\bar{B}_{M}\right)=\left\{\prod_{i, j=1}^{n} t_{i j}^{\ell N_{i j}} \mid N_{i j} \in \mathbb{N} \forall i, j\right\}$ is a $\mathbb{Z}_{\varepsilon}$-basis of $\mathfrak{F r}_{\mathbb{Z}}\left(\mathcal{O}^{\mathbb{Z}_{\varepsilon}}\left(M_{n}\right)\right)$. This last fact easily implies that $\mathrm{B}_{G L}^{M}$ is also $\mathcal{O}^{\mathbb{Z}_{\varepsilon}}\left(M_{n}\right)$ linearly independent, hence it is a basis of $\mathcal{O}_{\varepsilon}^{\mathbb{Z}_{\varepsilon}}\left(M_{n}\right)$ over $\mathcal{O}^{\mathbb{Z}_{\varepsilon}}\left(M_{n}\right)$ as claimed. 
As to $\mathcal{O}_{\varepsilon}^{\mathbb{Z}_{\varepsilon}}\left(G L_{n}\right)$, from definitions and the analysis in $\S 1$ we get (with $D_{\varepsilon}:=\left.D_{q}\right|_{\varepsilon}$ )

$$
\begin{aligned}
\mathcal{O}_{\varepsilon}^{\mathbb{Z}_{\varepsilon}}\left(G L_{n}\right) & =\mathcal{O}_{\varepsilon}^{\mathbb{Z}_{\varepsilon}}\left(M_{n}\right)\left[D_{\varepsilon}^{-1}\right]=\mathcal{O}_{\varepsilon}^{\mathbb{Z}_{\varepsilon}}\left(M_{n}\right)\left[D_{\varepsilon}^{-\ell}\right] \\
& =\mathcal{O}^{\mathbb{Z}_{\varepsilon}}\left(M_{n}\right)\left[D^{-1}\right] \bigotimes_{\mathcal{O}^{\mathbb{Z}_{\varepsilon}}\left(M_{n}\right)} \mathcal{O}_{\varepsilon}^{\mathbb{Z}_{\varepsilon}}\left(M_{n}\right)=\mathcal{O}^{\mathbb{Z}_{\varepsilon}}\left(G L_{n}\right) \bigotimes_{\mathcal{O}^{\mathbb{Z}_{\varepsilon}}\left(M_{n}\right)} \mathcal{O}_{\varepsilon}^{\mathbb{Z}_{\varepsilon}}\left(M_{n}\right)
\end{aligned}
$$

thus the result for $\mathcal{O}_{\varepsilon}^{\mathbb{Z}_{\varepsilon}}\left(G L_{n}\right)$ follows at once from that for $\mathcal{O}_{\varepsilon}^{\mathbb{Z}_{\varepsilon}}\left(M_{n}\right)$.

\section{Frobenius structures.}

3.1 Frobenius extensions and Nakayama automorphisms. Following [5], we say that a ring $R$ is a free Frobenius extension over a subring $S$, if $R$ is a free $S$-module of finite rank, and there is an isomorphism $F: R \longrightarrow \operatorname{Hom}_{S}(R, S)$ of $R-S$-bi-modules. Then $F$ provides a non-degenerate associative $S$-bilinear form $\mathbb{B}: R \times R \longrightarrow S$, via $\mathbb{B}(r, t)=F(t)(r)$. Conversely, one can characterise Frobenius extensions using such forms. When $S=\mathcal{Z}$ is contained in the centre of $R$, there is a $\mathcal{Z}$-algebra automorphism $v: R \longrightarrow R$, given by $r F(1)=F(1) v(r)$ (for all $r \in R$ ), and such $\mathbb{B}(x, y)=\mathbb{B}(v(y), x)$. This is called the Nakayama automorphism, and it is uniquely determined by the pair $\mathcal{Z} \subseteq R$, up to $\operatorname{Int}(R)$.

Proposition 3.2. (cf. [5], §2)

Let $R$ be a ring, $\mathcal{Z}$ an affine central subalgebra of $R$. Assume that $R$ is free of finite rank as a $\mathcal{Z}$-module, with a $\mathcal{Z}$-basis $\mathcal{B}$ that satisfies the following condition: there exists a $\mathcal{Z}$-linear functional $\Phi: R \rightarrow \mathcal{Z}$ such that for any non-zero $a=\sum_{b \in \mathcal{B}} z_{b} b \in R$ there exists $x \in R$ for which $\Phi(x a)=u z_{b}$ for some unit $u \in \mathcal{Z}$ and some non-zero $z_{b} \in \mathcal{Z}$.

Then $R$ is a free Frobenius extension of $\mathcal{Z}$. Moreover, for any maximal ideal $\mathfrak{m}$ of $\mathcal{Z}$, the finite dimensional quotient $R / \mathfrak{m} R$ is a finite dimensional Frobenius algebra.

This result is used in [5] to show that many families of algebras - in particular, some related to $\mathcal{O}_{\varepsilon}(G)$, where $G$ is a (complex, connected, simply-connected) semisimple affine algebraic group - are indeed free Frobenius extensions. But the authors could not prove the same for $\mathcal{O}_{\varepsilon}(G)$, as they did not know an explicit $\mathcal{O}(G)$-basis of $\mathcal{O}_{\varepsilon}(G)$. Now, following their strategy and using Theorem 2.3, I shall now prove that $\mathcal{O}_{\varepsilon}^{\mathbb{Z}_{\varepsilon}}(G)$ is free Frobenius over $\mathcal{O}^{\mathbb{Z}_{\varepsilon}}(G)$ when $G$ is $M_{n}$ or $G L_{n}$.

THeOREM 3.3. Let $G$ be $M_{n}$ or $G L_{n}$. Then $\mathcal{O}_{\varepsilon}^{\mathbb{Z}_{\varepsilon}}(G)$ is a free Frobenius extension of $\mathcal{O}^{\mathbb{Z}_{\varepsilon}}(G)$, with Nakayama automorphism $v$ given by $v\left(t_{i, j}\right)=\varepsilon^{2(i+j-n-1)} t_{i, j}(i, j=1, \ldots, n)$.

Proof. We prove that there is a suitable $\mathcal{O}^{\mathbb{Z}_{\varepsilon}}(G)$-linear functional $\Phi: \mathcal{O}_{\varepsilon}^{\mathbb{Z}_{\varepsilon}}(G) \longrightarrow$ $\mathcal{O}^{\mathbb{Z}_{\varepsilon}}(G)$ as required in Proposition 3.2, so that this result applies to $R:=\mathcal{O}_{\varepsilon}^{\mathbb{Z}_{\varepsilon}}(G)$ and $\mathcal{Z}:=\mathcal{O}^{\mathbb{Z}_{\varepsilon}}(G)$. by

Define $\Phi$ on the elements of the $\mathcal{O}^{\mathbb{Z}_{\varepsilon}}(G)$-basis $\mathrm{B}_{G L}^{M}$ of $\mathcal{O}_{\varepsilon}^{\mathbb{Z}_{\varepsilon}}(G)$ (see Theorem 2.3)

$$
\Phi\left(\prod_{i, j=1}^{n} t_{i, j}^{N_{i, j}}\right):=\prod_{i, j=1}^{n} \delta_{N_{i, j}, \ell-1}= \begin{cases}1, & \text { if } N_{i, j}=\ell-1 \forall i, j \\ 0, & \text { if not }\end{cases}
$$

(for all $0 \leq N_{i, j} \leq \ell-1$ ), and extend to all of $\mathcal{O}_{\varepsilon}^{\mathbb{Z}_{\varepsilon}}(G)$ by $\mathcal{O}^{\mathbb{Z}_{\varepsilon}}(G)$-linearity. In other words, $\Phi$ is the unique $\mathcal{O}^{\mathbb{Z}_{\varepsilon}}(G)$-valued linear functional on $\mathcal{O}_{\varepsilon}^{\mathbb{Z}_{\varepsilon}}(G)$ whose value is 1 on 
the basis element $\underline{t} \underline{\ell-1}:=\prod_{i, j=1}^{n} t_{i, j}^{\ell-1}$ and is zero on all other elements of the $\mathcal{O}^{\mathbb{Z}_{\varepsilon}}(G)$ basis $\mathrm{B}_{G L}^{M}$.

We claim that $\Phi$ satisfies the assumptions of Proposition 3.2, so the latter applies and proves our statement. Indeed, let us consider any non-zero $a=\sum_{\underline{t} \in \mathrm{B}_{G L}^{M}} z_{\underline{t}} \underline{t} \in$ $\mathcal{O}_{\varepsilon}^{\mathbb{Z}_{\varepsilon}}(G)$, and let $\underline{t}_{0}=\prod_{i, j=1}^{n} t_{i, j}^{N_{i, j}}$ in $\mathrm{B}_{G L}^{M}$ be such that $z_{t_{0}} \neq 0$ and $w\left(\underline{t}_{0}\right)$ is maximal (w.r.t. $\left.\leq_{l e x}\right)$. Then define $\underline{t}_{0}^{\vee}:=\prod_{i, j=1}^{n} t_{i, j}^{N_{i, j}^{\prime}}\left(\in \mathbb{B}_{G L}^{M}\right)$ with $N_{i, j}^{\prime}:=\ell-1-N_{i, j}$ for all $i, j=1, \ldots, n$. Quoting from the proof of Theorem 2.1(a), we know that $\underline{t}_{0}^{\vee} \underline{t}_{0}=$ $\varepsilon^{s} t \frac{\ell-1}{l}+$ l.t.'s, where $s \in \mathbb{Z}$ and the expression l.t.'s now stands for an $\mathcal{O}^{\mathbb{Z}_{\varepsilon}}(G)$-linear

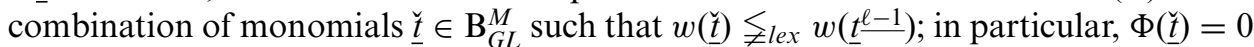
for all these $\underline{\underline{t}}$, hence eventually $\Phi\left(\underline{t}_{0}^{\vee} \underline{t}_{0}\right)=\varepsilon^{s} \Phi(\underline{t} \underline{\underline{\ell-1}})=\varepsilon^{s}$. Similarly, if $\underline{t}^{\prime} \in \mathbb{B}_{G L}^{M}$ is such that $w\left(\underline{t}^{\prime}\right)<_{\text {lex }} w(\underline{t})$, then $\underline{t}_{0}^{\vee} \underline{t}^{\prime}$ is an $\mathcal{O}^{\mathbb{Z}_{\varepsilon}}(G)$-linear combination of PBW monomials whose weight is at most $w\left(\underline{t}_{0}^{\vee} \underline{t}^{\prime}\right)$, hence $\Phi\left(\underline{t}_{0}^{\vee} \underline{t}^{\prime}\right)=0$. As we chose $\underline{t}_{0}$ so that $w\left(\underline{t}_{0}\right)$ is maximal, we eventually find

$$
\Phi\left(\underline{t}_{0}^{\vee} a\right)=\sum_{\underline{t} \in \mathrm{B}_{G L}^{M}} z_{\underline{t}} \Phi(\underline{t})=z_{\underline{t}_{0}} \Phi\left(\underline{t}_{0}\right)=\varepsilon^{s} z_{\underline{t}_{0}}
$$

where $\varepsilon^{s}$ is a unit in $\mathcal{O}^{\mathbb{Z}_{\varepsilon}}(G)$. So $\Phi$ satisfies the assumptions of Proposition 3.2, as claimed.

As to the Nakayama automorphism $v: \mathcal{O}_{\varepsilon}^{\mathbb{Z}_{\varepsilon}}(G) \longrightarrow \mathcal{O}_{\varepsilon}^{\mathbb{Z}_{\varepsilon}}(G)$, it is characterized (see $\S 3.1)$ by the property that $\mathbb{B}(x, y)=\mathbb{B}(v(y), x)$ for all $x, y \in R$. Here $\mathbb{B}$ is a $\mathcal{Z}$-bilinear form as in $\S 3.1$, which now is related to $\Phi$ by the formula $\mathbb{B}(x, y)=\Phi(x y)$ for all $x, y \in R$.

As $\Phi$ is an automorphism, and $\mathcal{O}_{\varepsilon}^{\mathbb{Z}_{\varepsilon}}(G)$ is generated - over $\mathcal{O}^{\mathbb{Z}_{\varepsilon}}(G)$ - by the $t_{i, j}$ 's, the claim about $v$ is proved if we show that

$$
\Phi\left(\prod_{r, s=1}^{n} t_{r, s}^{e_{r, s}} \cdot t_{i, j}\right)=\Phi\left(\varepsilon^{2(i+j-n-1)} t_{i, j} \cdot \prod_{r, s=1}^{n} t_{r, s}^{e_{r, s}}\right)
$$

Now, our usual argument shows that the expansions of the product of a generator $t_{i, j}$ and a PBW monomial $\prod_{r, s=1}^{n} t_{r, s}^{e_{r, s}}$ (in either order of the factors) as an $\mathcal{O}^{\mathbb{Z}_{\varepsilon}}(G)$-linear combination of elements of the $\mathcal{O}^{\mathbb{Z}_{\varepsilon}}(G)$-basis $\mathrm{B}_{G L}^{M}$ are of the form

$$
\begin{aligned}
& \prod_{r, s=1}^{n} t_{r, s}^{e_{r, s}} \cdot t_{i, j}=\varepsilon^{i+j-2 n} \prod_{r, s=1}^{n} t_{r, s}^{e_{r, s}+\delta_{r, i} \delta_{j, s}}+\text { l.t.'s } \\
& t_{i, j} \cdot \prod_{r, s=1}^{n} t_{r, s}^{e_{r, s}}=\varepsilon^{2-i-j} \prod_{r, s=1}^{n} t_{r, s}^{e_{r, s}+\delta_{r, i} \delta_{j, s}}+\text { l.t.'s. }
\end{aligned}
$$

This along with (3.1) gives

$$
\begin{aligned}
& \Phi\left(\prod_{r, s=1}^{n} t_{r, s}^{e_{r, s}} \cdot t_{i, j}\right)=\varepsilon^{i+j-2 n} \Phi\left(\prod_{r, s=1}^{n} t_{r, s}^{e_{r, s}+\delta_{r, i} \delta_{j, s}}\right)=\varepsilon^{i+j-2 n} \quad \text { if } e_{r, s}=\ell-1-\delta_{r, i} \delta_{j, s} \\
& \Phi\left(\prod_{r, s=1}^{n} t_{r, s}^{e_{r, s}} \cdot t_{i, j}\right)=\varepsilon^{i+j-2 n} \Phi\left(\prod_{r, s=1}^{n} t_{r, s}^{e_{r, s}+\delta_{r, i} \delta_{j, s}}\right)=0 \quad \text { if not }
\end{aligned}
$$


and similarly

$$
\begin{aligned}
& \Phi\left(t_{i, j} \cdot \prod_{r, s=1}^{n} t_{r, s}^{e_{r, s}}\right)=\varepsilon^{2-i-j} \Phi\left(\prod_{r, s=1}^{n} t_{r, s}^{e_{r, s}+\delta_{r, i} \delta_{j, s}}\right)=\varepsilon^{2-i-j} \quad \text { if } e_{r, s}=\ell-1-\delta_{r, i} \delta_{j, s} \\
& \Phi\left(t_{i, j} \cdot \prod_{r, s=1}^{n} t_{r, s}^{e_{r, s}}\right)=\varepsilon^{2-i-j} \Phi\left(\prod_{r, s=1}^{n} t_{r, s}^{e_{r, s}+\delta_{r, i} \delta_{j, s}}\right)=0 \quad \text { if not. }
\end{aligned}
$$

Direct comparison now shows that (3.2) holds, q.e.d.

AcKnowledgements. The author thanks I. Gordon, Z. Skoda, C. Stroppel and the referee for several useful comments.

\section{REFERENCES}

1. H. H. Andersen, W. Kexin and P. Polo, Representations of quantum algebras, Invent. Math. 104 (1991), 1-59.

2. G. M. Bergman, The diamond lemma for ring theory, Adv. Math. 29 (1978), 178-218.

3. K. A. Brown and I. Gordon, The ramifications of the centres: quantised function algebras at roots of unity, Proc. London Math. Soc. (3) 84 (2002), 147-178.

4. K. A. Brown, I. Gordon and J. T. Stafford, $\mathcal{O}_{\varepsilon}[G]$ is a free module over $\mathcal{O}[G]$ preprint http://arxiv.org/abs/math.QA/0007179 (2000), 3 pages.

5. K. A. Brown, I. Gordon and C. Stroppel, Cherednik, Hecke and quantum algebras as free modules and Calabi-Yau extensions preprint http://arxiv.org/abs/math.RT/0607170 (2006), 31 pages. 1994).

6. V. Chari and A. Pressley, A guide to quantum groups (Cambridge University Press

7. C. De Concini and V. Lyubashenko, Quantum function algebra at roots of 1, Adv. Math. 108 (1994), 205-262.

8. L. Dạbrowski, C. Reina and A. Zampa, $A\left(\mathrm{SL}_{q}(2)\right)$ at roots of unity is a free module over $A(\mathrm{SL}(2))$ Lett. Math. Phys. 52 (2000), 339-342.

9. F. Gavarini, Quantum function algebras as quantum enveloping algebras, Comm. Algebra 26 (1998), 1795-1818.

10. H. T. Koelink, On $*$-representations of the Hopf $*$-algebra associated with the quantum group $U_{q}(n)$, Compositio Math. 77 (1992), 199-231.

11. T. Levasseur and J. T. Stafford, The quantum coordinate ring of the special linear group, J. Pure Appl. Algebra 86 (1993), 181-186. 439.

12. B. Parshall and J. Wang, Quantum linear groups, Mem. Amer. Math. Soc. 89 (1991), no. 